Supporting information

for

\title{
Chiral Recognition in Cucurbituril Cavities
}

\author{
Mikhail V. Rekharsky, ${ }^{\dagger}$ Hatsuo Yamamura, ${ }^{\ddagger}$ Chika Inoue, ${ }^{\ddagger}$ Masao Kawai, ${ }^{\ddagger}$ Issey Osaka, \\ Ryuichi Arakawa, ${ }^{\Uparrow}$ Kouhei Shiba, ${ }^{\dagger \dagger}$ Akihiro Sato, ${ }^{\dagger}$ Young Ho Ko, ${ }^{\S}$ Narayanan \\ Selvapalam, ${ }^{\S}$ Kimoon Kim, ${ }^{*}$, and Yoshihisa Inoue $*, \dagger$ \\ ${ }^{\dagger}$ Entropy Control Project, ICORP, JST, 4-6-3 Kamishinden, Toyonaka 560-0085, Japan, \\ *Graduate School of Technology, Nagoya Institute of Technology, Gokiso-cho, Showa-ku \\ Nagoya 466-8555, Japan, "Department of Applied Chemistry, Kansai University, Yamatecho, \\ Suita 564-0073, Japan, ${ }^{\dagger S}$ Scientific Instrumentation Division, Sysmex Co, 4-4-4 Takatsuka-dai, \\ Nishi-ku, Kobe 651-2271, Japan, " JASCO International Co., 1-11-10 Myojin-cho, Hachioji \\ 192-0046, Japan, and ${ }^{\S}$ National Creative Research Initiative Center for Smart Supramolecules \\ and Department of Chemistry, Division of Molecular and Life Sciences, Pohang University of \\ Science and Technology, San 31 Hyojadong, Pohang 790-784, Korea
}

e-mail: inoue@chem.eng.osaka-u.ac.jp 


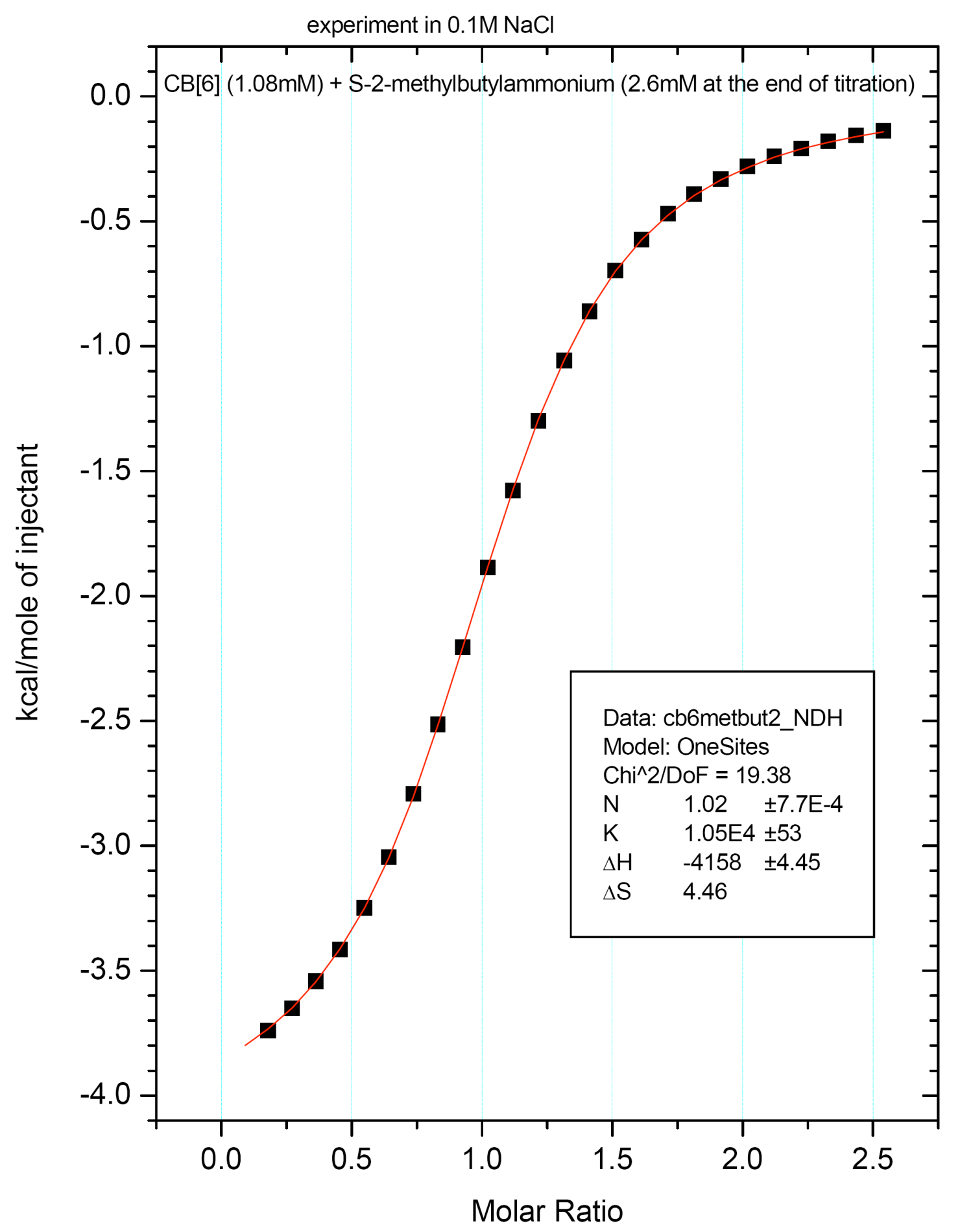

Figure SI-1. Interaction of $(S)$-2-methylbutylammonium cation with $\mathrm{CB}[6]$ in aqueous $0.1 \mathrm{M}$ $\mathrm{NaCl}$. 
a)

b)

c)

d)
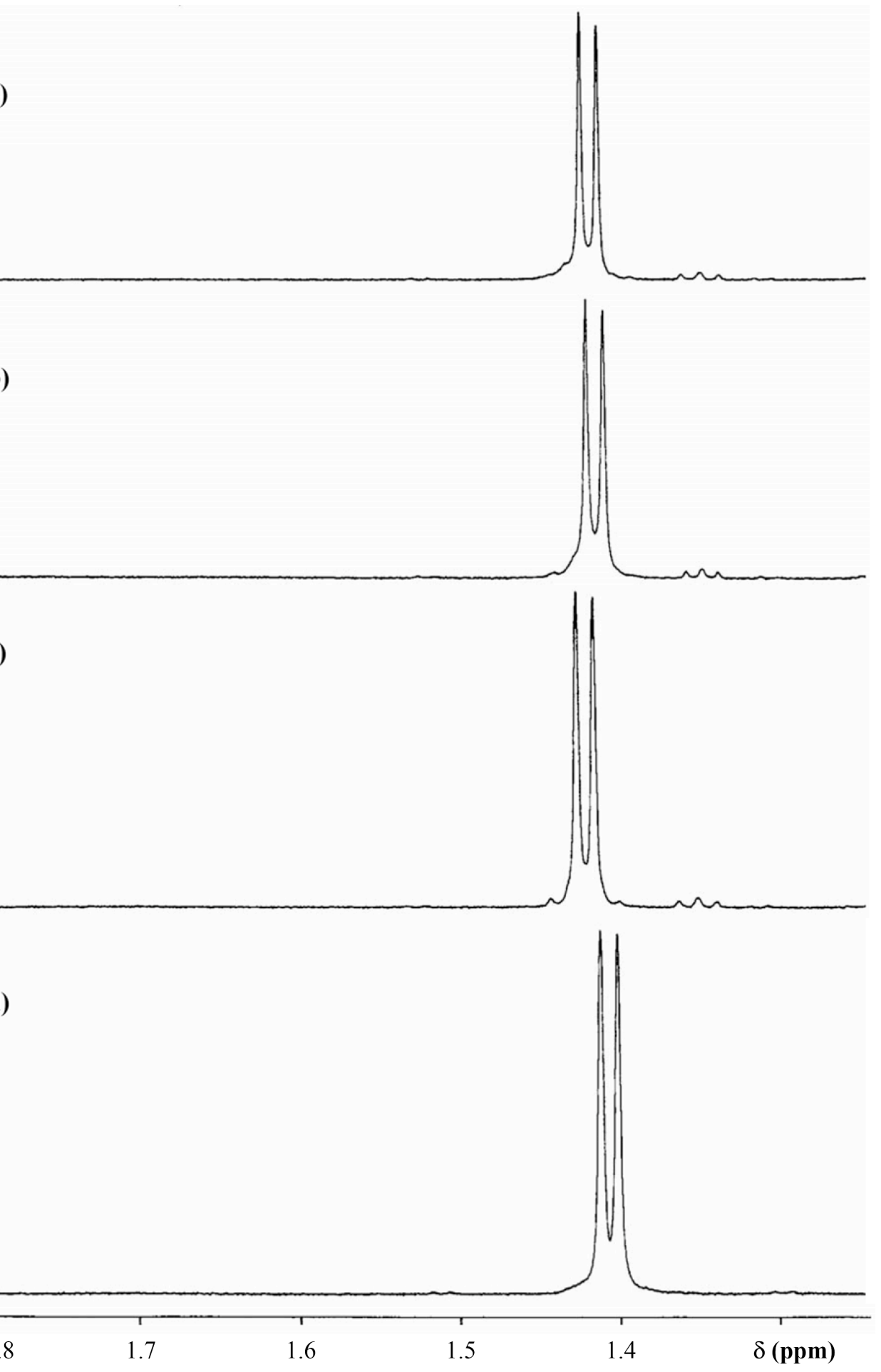

Figure SI-2. The methyl proton signals of 2-methylpiperazine in the NMR spectra of $\mathrm{D}_{2} \mathrm{O}$ solutions of (a) $5 \mathrm{mM}(R)-2$-methylpiperazine $2 \mathrm{HCl}$, (b) $0.5 \mathrm{mM} \mathrm{CB}[6]+5 \mathrm{mM}(R)-2$ methylpiperazine $2 \mathrm{HCl}$, (c) $0.5 \mathrm{mM} \mathrm{CB}[6]+0.5 \mathrm{mM}(S)$-2-methylbutylamine $+5 \mathrm{mM}(R)$-2methylpiperazine $2 \mathrm{HCl}$, and (d) $0.5 \mathrm{mM} \mathrm{CB}[6]+0.5 \mathrm{mM}(S)$-2-methylbutylamine + $5 \mathrm{mM}(S)$-2methylpiperazine $2 \mathrm{HCl}$. 
CB6 $(0.5 \mathrm{mM})+\mathrm{S}-2$-metylbutylamine $(0.1 \mathrm{mM})+\mathrm{R} / \mathrm{S}-2$ methylpiperazine $(5 \mathrm{mM})$ in $10.1 \mathrm{mMaq} \cdot \mathrm{HCl}$

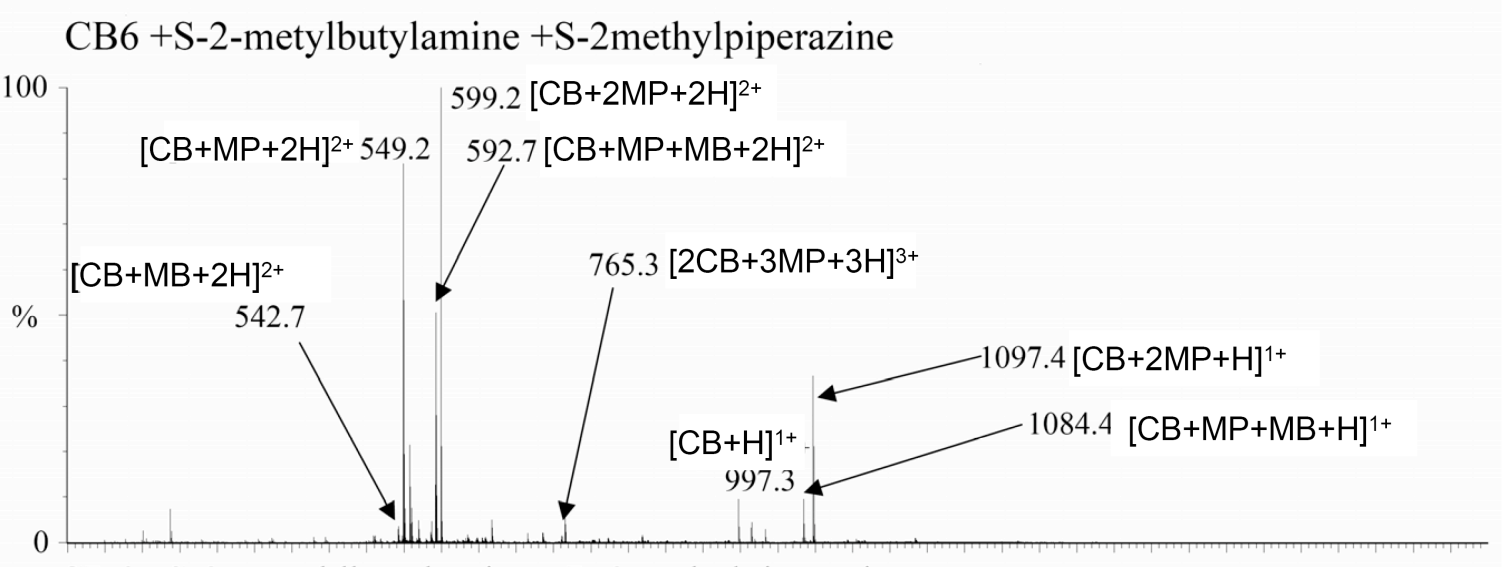

CB6 +S-2-metyhlbutylamine +R-2methylpiperazine

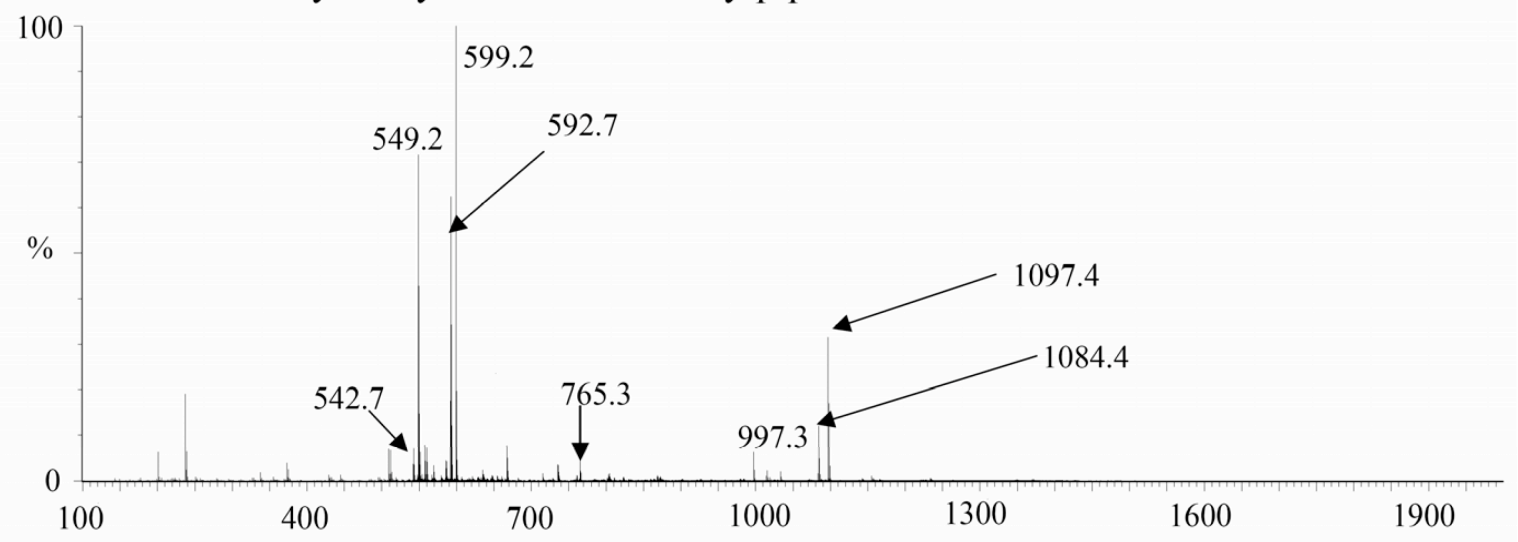

Figure SI-3. ESI-MS spectra of an aqueous three-component solution containing $0.5 \mathrm{mM} \mathrm{CB}[6]$, $5 \mathrm{mM}(R) /(S)$-2-methylpiperazine, and $0.1 \mathrm{mM}(S)$-2-methylbutylamine. 


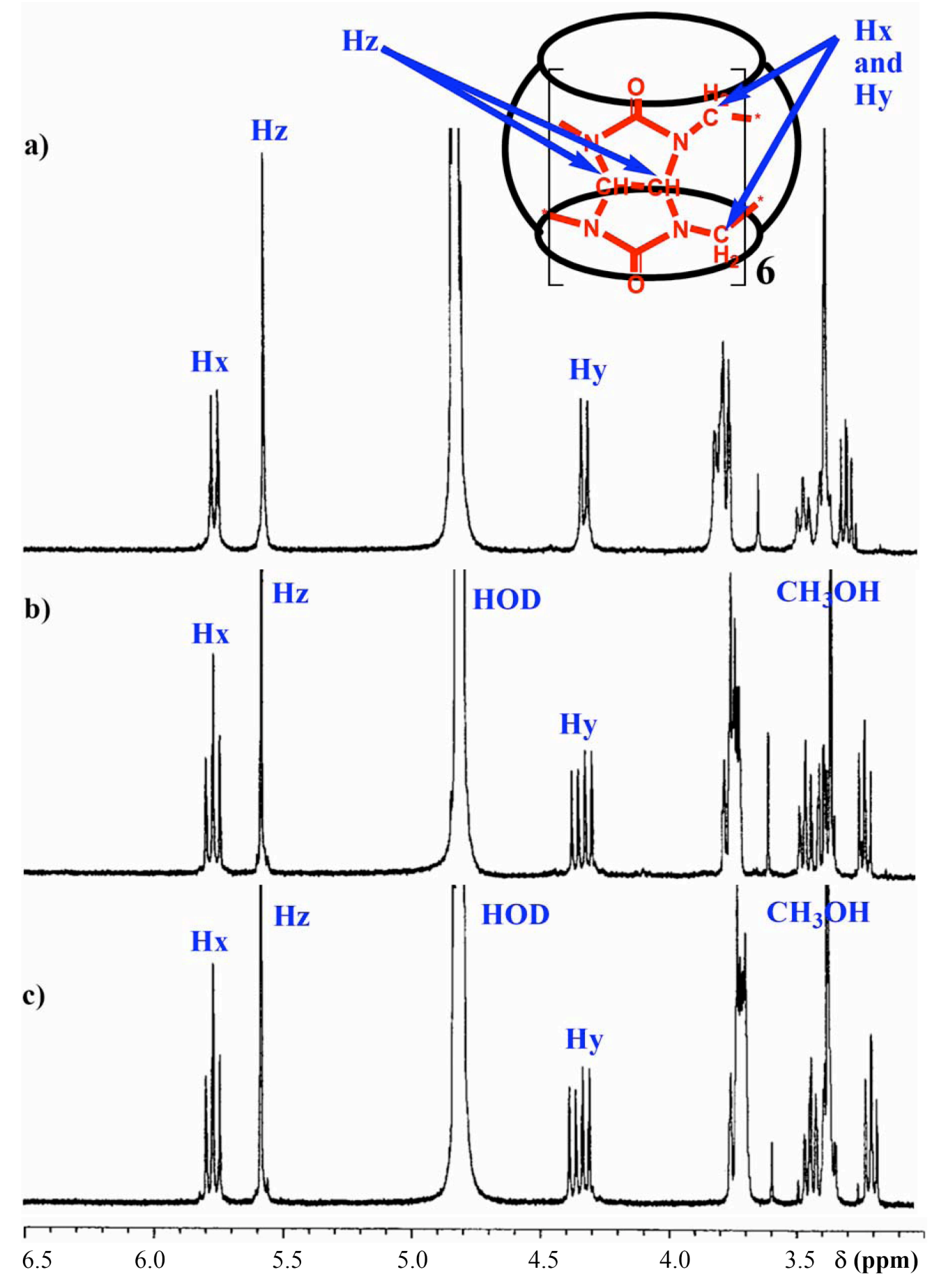

Figure SI-4. NMR spectra of $\mathrm{D}_{2} \mathrm{O}$ solutions of (a) $0.5 \mathrm{mM} \mathrm{CB}[6]+5 \mathrm{mM}(R)-2$ methylpiperazine $2 \mathrm{HCl}$, (b) $0.5 \mathrm{mM} \mathrm{CB}[6]+5 \mathrm{mM}(R)$-2-methylpiperazine $2 \mathrm{HCl}+0.5 \mathrm{mM}(S)$ 2-methylbutylamine $\cdot \mathrm{HCl}$, and (c) $0.5 \mathrm{mM} \mathrm{CB}[6]+5 \mathrm{mM}(S)$-2-methylpiperazine $\bullet 2 \mathrm{HCl}+0.5$ $\mathrm{mM}(S)$-2-methylbutylamine $\cdot \mathrm{HCl}$. 

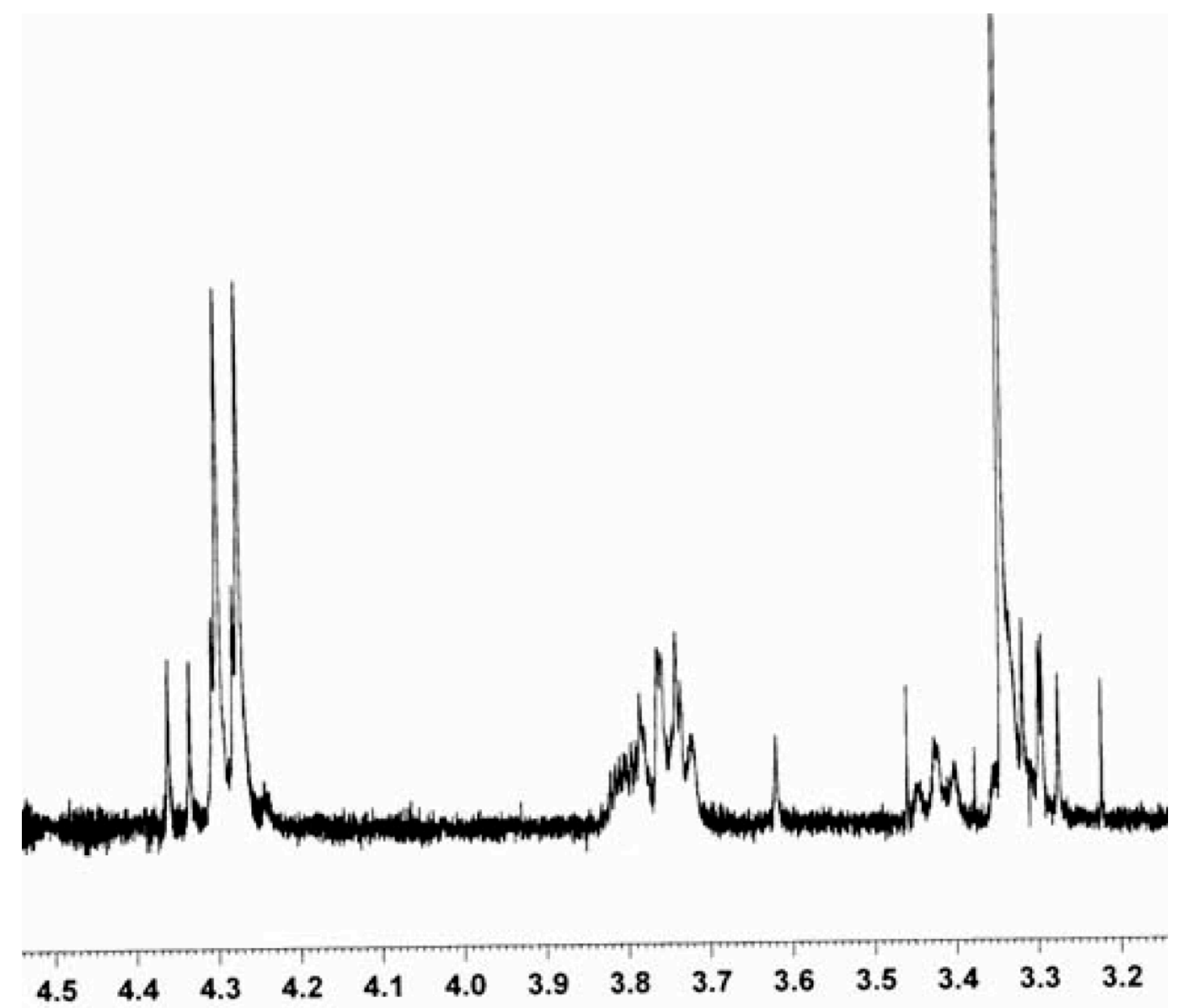

Figure SI-5. NMR spectrum of a mixture of diastereomeric complexes $(0.5 \mathrm{mM} \mathrm{CB}[6]+0.1 \mathrm{mM}$ $(S)$-methylbutylamine $\cdot \mathrm{HCl}+6 \mathrm{mM} \quad(R)$-2-methylpiperazine $2 \mathrm{HCl}+5 \mathrm{mM} \quad(S)$-2methylpiperazine $2 \mathrm{HCl}$. 
ROESY spectra of Phe-D-Leu-NH2 and that with CB7.

$[$ Phe-D-Leu-NH2 HCl] $=5 \mathrm{mM},[\mathrm{CB} 7\}=5 \mathrm{mM}, 0.1 \mathrm{M} \mathrm{NaCl}-\mathrm{D} 2 \mathrm{O}$
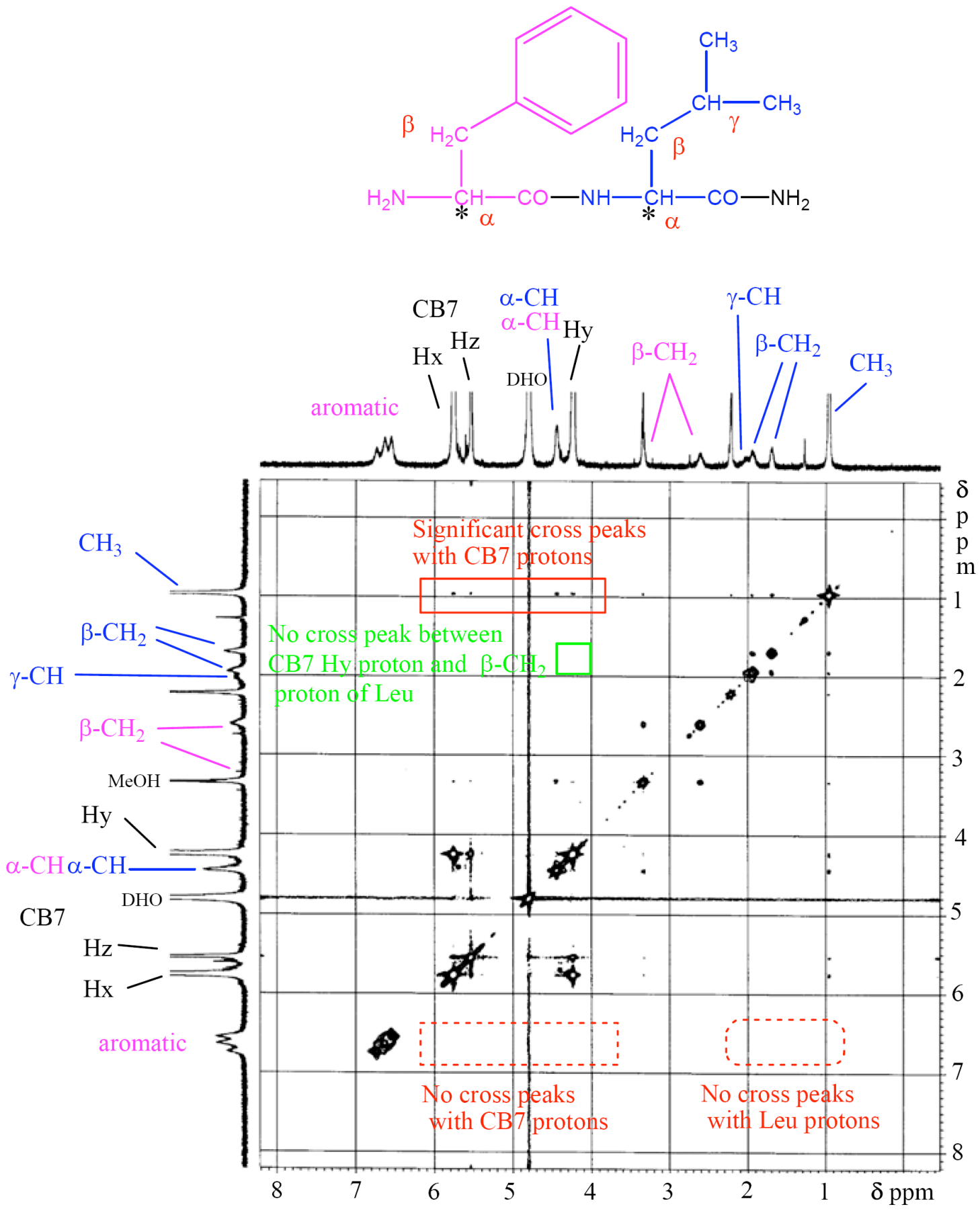

Figure SI-6. ROESY spectra of L-Phe-D-Leu-NH $\mathrm{NH}_{2}+\mathrm{CB}$ [7] complex. 


\section{ROESY spectra of Phe-L-Leu-NH2 and that with CB7.}

[Phe-L-Leu-NH2 HBr] $=5 \mathrm{mM},[\mathrm{CB} 7\}=5 \mathrm{mM}, 0.1 \mathrm{M} \mathrm{NaCl}-\mathrm{D} 2 \mathrm{O}$
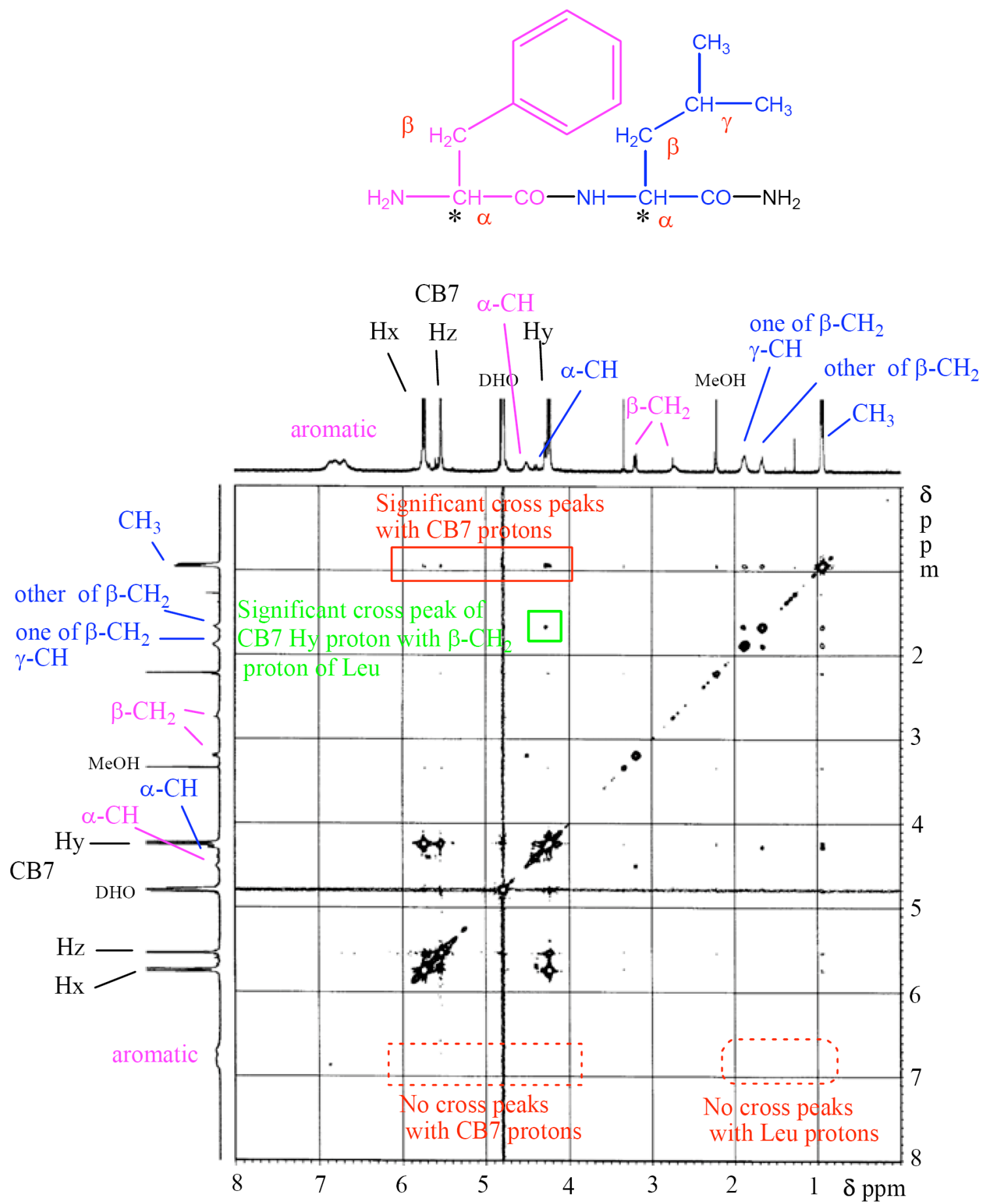

Figure SI-7. ROESY spectra of L-Phe-L-Leu-NH $\mathrm{N}_{2}+\mathrm{CB}[7]$ complex. 